\title{
Plasma rotation and ion temperature measurements by collective Thomson scattering at ASDEX Upgrade
}

\author{
M. Stejner ${ }^{1}$, S. K. Nielsen ${ }^{1}$, A. S. Jacobsen ${ }^{1}$, S. B. Korsholm ${ }^{1}$, \\ F. Leipold ${ }^{1}$, R. M. McDermott ${ }^{2}$, P. K. Michelsen ${ }^{1}$, \\ J. Rasmussen ${ }^{1}$, M. Salewski ${ }^{1}$, M. Schubert ${ }^{2}$, J. Stober ${ }^{2}$, \\ D. H. Wagner ${ }^{2}$, and the ASDEX Upgrade Team \\ ${ }^{1}$ Technical University of Denmark, Department of Physics, Kgs. Lyngby, Denmark \\ ${ }^{2}$ Max-Planck-Institut für Plasmaphysik, Boltzmannstr. 2, Garching, Germany \\ E-mail: mspe@fysik.dtu.dk
}

\begin{abstract}
We present the first deuterium ion temperature and rotation measurements by collective Thomson scattering at ASDEX Upgrade. The results are in general agreement with boron-based charge exchange recombination spectroscopy measurements and consistent with neoclassical simulations for the plasma scenario studied here. This demonstration opens the prospect for direct non-perturbative measurements of the properties of the main ion species in the plasma core with applications in plasma transport and confinement studies.
\end{abstract}

23 February 2015

PACS numbers: 52.25.Os, 52.35.Hr, 52.40.Db, 52.70.Gw, 52.70.-m

\section{Introduction}

We here present the first main ion temperature and rotation measurements by collective Thomson scattering (CTS) on ASDEX Upgrade (AUG). Knowledge of ion temperatures and plasma rotation velocities, essential to understand the confinement and fusion performance of tokamak plasmas, is commonly inferred from spectroscopic measurements of impurities. However, substantial differences between deuterium and impurity ion rotation velocities have recently been observed at levels beyond neoclassical predictions for certain plasma conditions in DIII-D [1]. Main ion measurements are therefore highly relevant and may even affect predictions of the performance of future fusion reactors such as ITER [2].

CTS measurements provide information about the ion velocity distribution in tokamak plasmas while imparting no significant energy or momentum to the plasma [3]. CTS diagnostics are based on measurements of electromagnetic probing radiation scattered off microscopic plasma fluctuations arising mainly due to electron screening 
of ions $[3,4]$. The spectra are therefore sensitive to the ion velocity distribution, and properties of the bulk ions can be inferred by fitting the thermal ion feature at relatively low frequency shifts with a model for CTS. Microwave based CTS is also a robust diagnostic able to operate under reactor relevant conditions and to access the core of large burning fusion plasmas [5]. In addition to its role as a fast-ion diagnostic [6,7], CTS is therefore also considered a potentially attractive technique for measurements of bulk-ion parameters such as plasma rotation and ion temperature [8-10]. As a nonperturbative diagnostic measuring the properties of the main ion species, CTS is well suited for this role and can complement more standard diagnostics and access new experimental regimes.

The CTS receiver system at ASDEX Upgrade (AUG) was recently expanded to enable detailed resolution of the thermal ion feature of the CTS spectra [11], and on this basis we show here the first bulk-ion temperature and plasma rotation measurements by CTS in AUG. The CTS measurements are found to agree well with boron-based active charge exchange recombination spectroscopy (CXRS) measurements, which is consistent with neoclassical simulations predicting only small differences between deuterium and boron temperatures and velocities for the plasma scenario studied here.

\section{Measurement technique}

On AUG [12], the probing radiation is delivered by one of the four dual-frequency gyrotrons otherwise used for electron cyclotron resonance heating $(\mathrm{ECRH})$ in the ECRH II system [13]. The two CTS receiver systems view the plasma using the launching antennae and quasi-optical transmission lines of gyrotrons 1 and 2 while gyrotron 3 delivers the $105 \mathrm{GHz}$ probing radiation with a power of $500 \mathrm{~kW}$; see Fig. 1 for an illustration of the beam geometry. To enable background subtraction, the probe power was modulated on/off in a duty cycle with $2 \mathrm{~ms}$ on-periods and $8 \mathrm{~ms}$ off-periods (except during the interval $t=2.05$ to $2.15 \mathrm{~s}$ when the off-period was $4 \mathrm{~ms}$ ). The receiver mirrors were oriented to give parallel viewing beams, one of which intersected the probing beam to pick up scattered radiation. The other receiver was used for improved background subtraction $[11,14]$.

At the magnetic field used here, the fundamental electron cyclotron resonance for the probing frequency was slightly inside the plasma on the high-field side (see Fig. 1). This results in a first-pass absorption rate of around $10 \%$. Considering also the low duty cycle (20\% during most of the discharge), we do not expect this small amount of absorption to cause any significant perturbation to the plasma, and experimentally we have seen no evidence of any perceptible perturbation to the density, temperature or rotation rates.

The CTS measurement volume was centered on $(R, z)=(1.73 \mathrm{~m},-0.11 \mathrm{~m})$ at a normalized poloidal flux coordinate of 0.36 . The orientation of the probe and receiver mirrors determine the direction for the wave vectors of the incident, $\mathbf{k}^{\mathrm{i}}$, and received scattered, $\mathbf{k}^{\mathrm{s}}$, radiation. The difference, $\mathbf{k}^{\delta}=\mathbf{k}^{\mathrm{s}}-\mathbf{k}^{\mathrm{i}}$, determines the wave vector of 

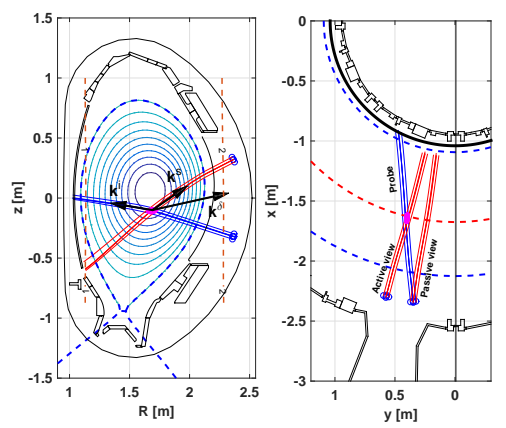

Figure 1. Left: a poloidal projection of the CTS beam geometry in AUG discharge 29600. Magnetic flux surfaces are indicated by the nested contours, and the vertical dashed lines show the locations of the fundamental and second harmonic electron cyclotron resonances for the probing frequency. Beam patterns were calculated by ray tracing. The CTS measurement was localized in the volume defined by the beam overlap indicated by the magenta ellipsoid. CTS resolves fluctuations with wave vectors $\mathbf{k}^{\delta}=\mathbf{k}^{s}-\mathbf{k}^{i}$ as indicated by the black arrows (not to scale). Right: a top-view showing a toroidal projection of the beam geometry.

the resolved plasma fluctuations. CTS measurements are sensitive to a 1D-projection of the ion velocity distribution onto $\mathbf{k}^{\delta}$, and plasma rotation then causes a Doppler shift in the CTS spectrum of $\mathbf{v}_{i} \cdot \mathbf{k}^{\delta} / 2 \pi$, where $\mathbf{v}_{i}$ is the rotation velocity. The present experiments were conducted with a scattering angle $\angle\left(\mathbf{k}^{\mathrm{i}}, \mathbf{k}^{\mathrm{s}}\right)=122^{\circ}$ and a projection angle $\angle\left(\mathbf{k}^{\delta}, \mathbf{B}\right)=106^{\circ}$.

The CTS receivers are essentially highly sensitive heterodyne radiometers. The thermal ion feature is resolved using a recently installed fast acquisition system enabling direct digitization (at 6.25 GSamples/s) and Fourier analysis of the time-domain signal in the relevant frequency range. We used an FFT-window of $2^{12}$ samples giving a frequency resolution of $1.53 \mathrm{MHz}$ within about $\pm 1 \mathrm{GHz}$ of the probing frequency. The CTS receivers are protected against stray probing radiation by notch filters. Within the stop bands of these filters, effectively from $120 \mathrm{MHz}$ below the probing frequency to $190 \mathrm{MHz}$ above, the signal is suppressed and also cannot be calibrated. That frequency range will hence be excluded in the analysis presented below. The design, calibration and operation of the fast receiver system were recently described at length elsewhere [11].

\section{Measurement results and analysis}

The measurements presented here were obtained in AUG discharge 29600 with a plasma current of $800 \mathrm{kA}$ and on-axis magnetic field strength of $-2.6 \mathrm{~T}$. The plasma consisted mainly of deuterium and was heated with 0.4-0.9 MW of $140 \mathrm{GHz}$ central ECRH. In addition, the 2.5 MW NBI sources Q3 (60 keV) and Q8 (93 keV) were alternated and combined to vary the NBI heating power. The bulk-ion related CTS measurements were timed to cover the NBI heated phases and to extend across the NBI turn-offs.

Figure 2 shows a spectrogram of the CTS measurements as well as time traces for the 


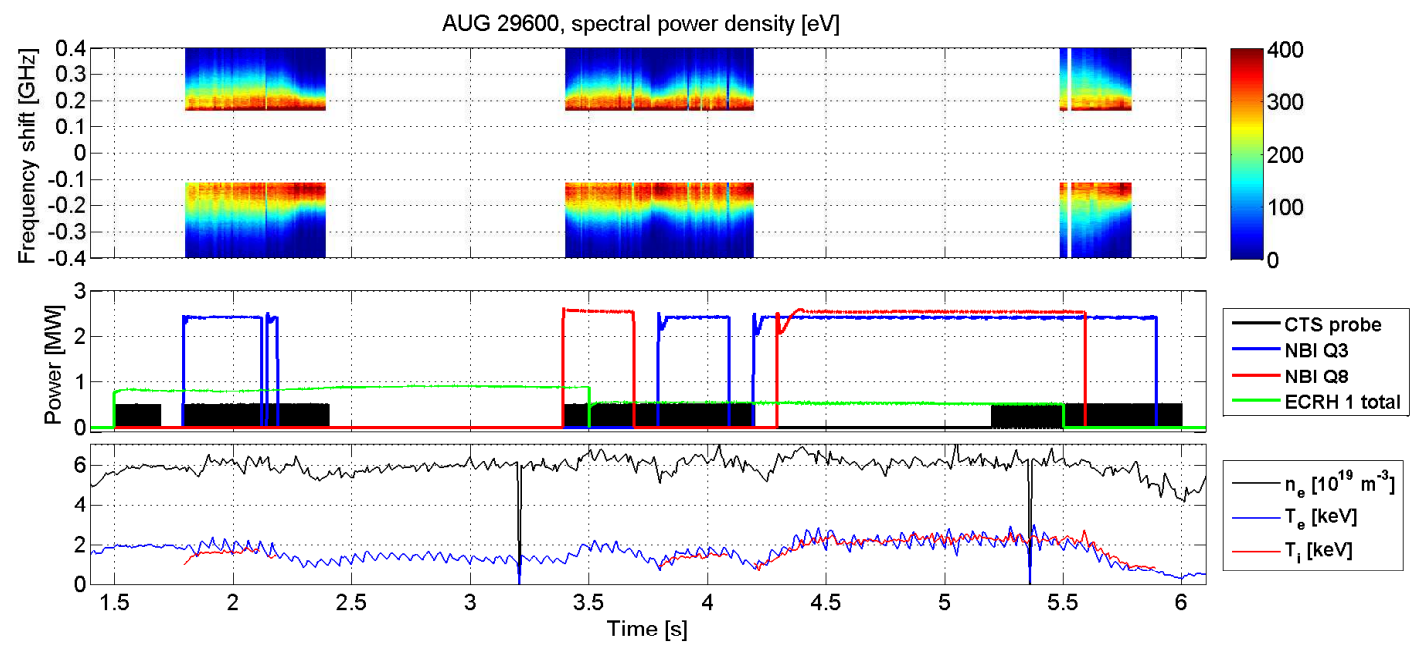

Figure 2. Upper panel: a spectrogram showing the bulk-ion region of the CTS spectra obtained in AUG discharge 29600. The notch filter stop band is excluded. Middle panel: heating timetraces and the CTS probe power. Lower panel: the electron density and temperature from incoherent Thomson scattering and the ion temperature from CXRS, all mapped to the CTS measurement volume.

probe and heating powers. The lower panel shows density and temperature measurement by incoherent Thomson scattering and CXRS, all interpolated to the magnetic flux coordinate of the CTS measurement. The CXRS measurements were based on charge exchange reactions between boron impurities and neutral deuterium originating from NBI Q3. The boron ion temperature from CXRS is therefore shown only for periods when Q3 was on. The electron density stayed roughly constant around $6 \times 10^{19} \mathrm{~m}^{-3}$. The electron and ion temperatures varied between 0.9 and $2.6 \mathrm{keV}$ depending on the NBI heating. The CTS spectra in the upper panel show a clear response to the variations in NBI heating demonstrating sensitivity to the resulting changes in plasma temperature. As expected, the spectra become wider at higher heating power and temperature and vice versa at lower temperature. The Doppler shift due to rotation is more difficult to distinguish on this scale.

In order to interpret the CTS measurements, we fit the measured spectra with a model for CTS following the approach in previous applications of CTS to bulk- and fast-ion measurements on AUG [6] and TEXTOR [7, 10]. The model describes the plasma fluctuations within a kinetic and fully electromagnetic theory and also treats the scattering process itself in a fully electromagnetic approach [4]. We assume a deuterium dominated plasma and include a number of impurity ion species $\left({ }^{4} \mathrm{He},{ }^{12} \mathrm{C},{ }^{56} \mathrm{Fe}\right.$ and ${ }^{184} \mathrm{~W}$ ) assumed to be in thermal equilibrium with the bulk ions. The impurities affect the spectral shape mainly at low frequency shifts in or near the notch filter stop band. Effects of fast ions are not included as they are negligible compared to bulk ions in the frequency range considered here. The model thus assumes Maxwellian velocity distributions with plasma rotation described as a drift velocity parallel to the magnetic field. For comparison with CXRS, the drift velocity inferred from the fits should 
therefore be projected on the toroidal rotation velocity measured by CXRS. However, for the scattering geometry used here, the magnetic field in the CTS measurement volume was very nearly toroidal, and the projection therefore amounts to a negligible correction of about $0.3 \%$.

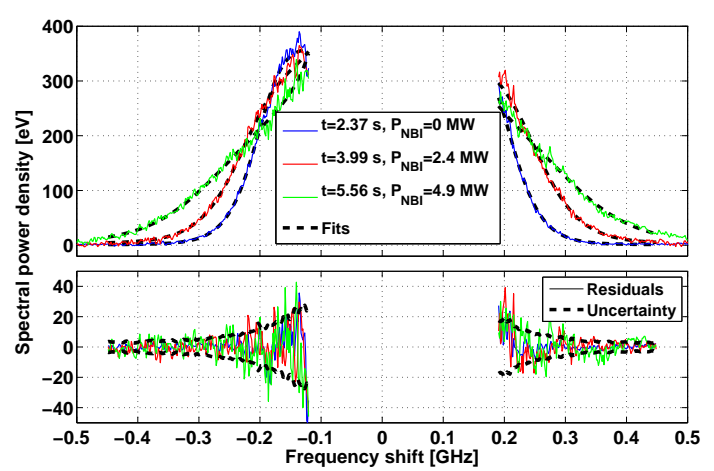

Figure 3. Upper panel: examples of measured and fitted CTS spectra from AUG discharge 29600 at different NBI heating powers. Lower panel: Residuals and the mean uncertainty for the three spectra shown above (plus/minus one standard deviation).

The fits were performed within the framework of a least squares method of inference [15]. The deuterium ion temperature and drift velocity were considered free fitting parameters with large initial uncertainty (i.e. broad Bayesian priors) and constant starting points for the optimization $\left(1.5 \mathrm{keV}\right.$ and $10^{4} \mathrm{~m} / \mathrm{s}$, respectively). So, to permit independent comparison, no prior information from other diagnostics was included for the bulk-ion parameters. The impurity densities were initially assumed to constitute small fractions of the electron density, $10^{-3}$ in all cases except ${ }^{4} \mathrm{He}$ at $3 \times 10^{-2}$, consistent with spectroscopic measurements, and they remain in that range in the final fits. The electron temperature and density were obtained from incoherent Thomson scattering measurements mapped to the CTS measurement location and were treated as fixed parameters. Values for the local magnetic field and the angles parameterizing the scattering geometry were obtained from magnetic equilibrium reconstruction and ray tracing. These were also treated as fixed parameters. Prior uncertainties were estimated from the level of temporal fluctuations in the relevant data. The fits further account for uncertainties in the CTS spectra themselves, which are similarly estimated from the level of temporal fluctuations in each frequency bin. The final uncertainties on the parameters of interest (here ion temperature and rotation) are calculated using a Bayesian formalism from the width of the posterior probability distribution [15]. They are therefore not simply given by the frequency resolution of the spectra but rather account for uncertainties in both the CTS measurements and the prior information on all model parameters as well as for the detailed functional dependence of the CTS spectra on both plasma and system parameters.

Figure 3 shows three examples of fits to CTS spectra measured for different NBI heating powers. The spectral width clearly varies with the NBI heating power and close inspection also shows a Doppler shift due to plasma rotation during NBI heating. The 
fitted theoretical spectra (thick dashed lines) reproduce the measured spectra well with residuals below or near the statistical uncertainty in the measurements.

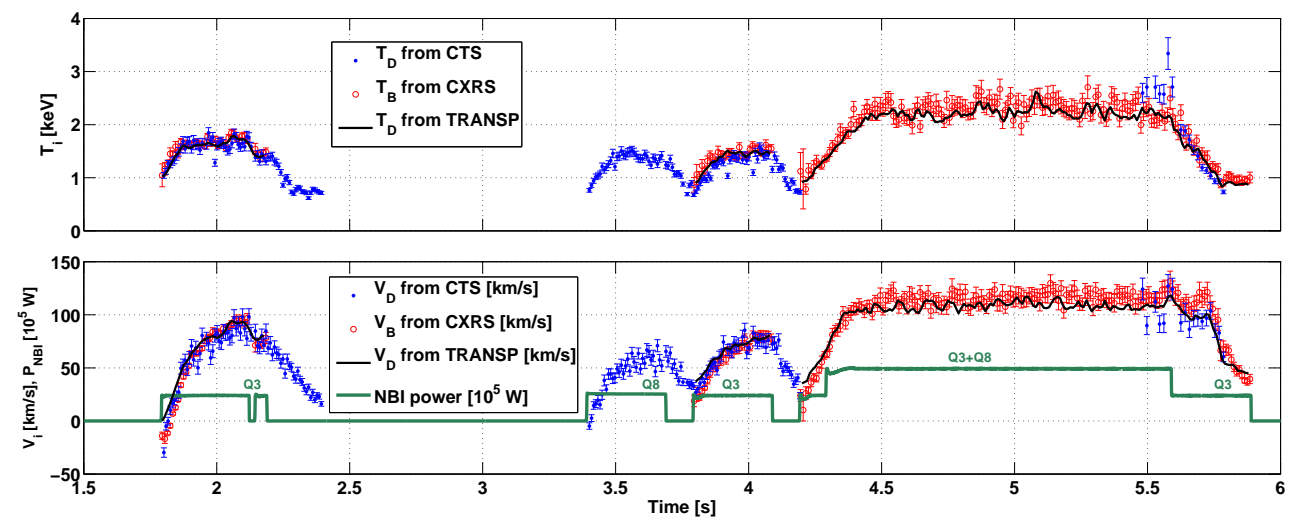

Figure 4. Deuterium ion temperatures (upper panel) and plasma rotation velocities (lower panel) inferred from theoretical fits to the measured CTS spectra and compared to TRANSP results for deuterium and the boron-based CXRS measurements mapped to the same flux coordinate. Here positive velocities correspond to co-current (counter$B_{t}$ ) rotation. The error bars indicate plus/minus one standard deviation of the statistical uncertainty in both measurements. The lower panel further shows the total NBI power.

Figure 4 shows the inferred ion temperature (upper panel) and plasma rotation (lower panel). The lower panel further shows a time trace of the total NBI heating power, and the CTS results are compared with CXRS measurements and TRANSP results. The CTS measurements clearly capture the plasma response to the changing heating power. The plasma is seen to heat and spin up as each NBI source is turned on and vice versa during non-NBI heated phases. We also note that the rotation reversal at $t=1.8 \mathrm{~s}$, just after the NBI Q3 is first turned on, is measured by CTS as well as CXRS. Uncertainties on the inferred bulk ion parameters are in the ranges of $3-10 \mathrm{~km} / \mathrm{s}$ and $30-200 \mathrm{eV}$, or on average about $10 \%$ in the rotation velocity and about $5 \%$ in the ion temperature.

\section{Discussion}

Within their uncertainties, the CTS and CXRS measurements are generally in good agreement except for some outliers during and just after the phase with $P_{\mathrm{NBI}} \simeq 5 \mathrm{MW}$. Differences could in principle arise due to the different ion species and measurement locations. Simulations with the SLVTX and NCLASS modules in TRANSP $[16,17]$ were used to estimate the expected size of such differences. Results for the deuterium ions are shown in Figure 4 for the periods when CXRS was available to constrain the simulation. We here assume the temperature to be a flux function and interpolate the CXRS and TRANSP results to the flux coordinate of the CTS measurements. The simulated boron temperature differs by less than $3 \%$ from that of deuterium and is 
not shown. The comparison is less straightforward for the rotation since it is not a flux function. However, for each species fluid, the rotation can be described as an incompressible flow tangential to flux surfaces [18]. In Figure 4 we used this to map the velocities calculated with TRANSP at the magnetic midplane to the CTS measurement location. No such mapping was possible for the CXRS measurements, which are shown simply for the same flux coordinate. However, we note that the simulated boron rotation velocity at the CXRS measurement location differs by less than $10 \%$ from the simulated deuterium velocity at the CTS measurement location, i.e. within the uncertainty of the measurements. The general agreement between the CTS and CXRS measurements is thus consistent with expectations based on the TRANSP simulation.

\section{Conclusions and outlook}

We have presented the first bulk-ion measurements by CTS on AUG building on new acquisition techniques enabling the thermal ion feature of the CTS spectra to be resolved in detail. Results for the deuterium ion temperature and plasma rotation velocity were in general agreement with boron-based CXRS measurements which is consistent with neoclassical simulations using TRANSP. The statistical uncertainty amounted on average to $5 \%$ in the ion temperature and $10 \%$ in the rotation velocity giving a signalto-noise ratio sufficient to resolve the plasma response to variations in the NBI heating.

A systematic evaluation of CTS as a bulk-ion diagnostic would be required to fully assess the diagnostic potential and optimum plasma/system configuration for this technique, but we may here note that bulk-ion measurements by CTS may complement the more standard CXRS technique and have certain useful properties worth mentioning at this stage. First, CTS measurements do not rely on NBI heating and impart little to no energy or momentum to the plasma provided the magnetic field is chosen appropriately. CTS is thus well suited for measurements in Ohmic or low powered plasmas, e.g. for studies of intrinsic rotation reversals where it is important to avoid perturbations to the plasma. Second, CTS measures the properties of the main ion species rather than those of impurities. No major differences were expected or observed between the main and impurity ions for the NBI heated plasma studied here (making this a useful test case for our present purpose). However, substantial differences, inconsistent with both the sign and magnitude of neoclassical predictions, have been observed in DIIID, e.g. for the intrinsic rotation in ECRH dominated H-modes [1]. Notably, this could affect the expected collisionality scaling for the rotation and thus the radial electric field which plays a key role in stabilizing turbulence and improving plasma confinement [2].

We finally note that a $60 \mathrm{GHz}$ CTS system is currently being designed for ITER. This system will mainly be oriented towards fast-ion velocity distribution measurements, but it is also expected to act in a supplementary role as a bulk-ion diagnostic [5]. The results presented here demonstrate the techniques required for such measurements, and the experience gained on AUG may well influence the design of the ITER CTS system. 


\section{Acknowledgments}

This project has received funding from the European Union's Horizon 2020 research and innovation programme under grant agreement number 633053. The views and opinions expressed herein do not necessarily reflect those of the European Commission.

\section{References}

[1] B. A. Grierson, et al. Physics of Plasmas, 19(5):056107, March 2012.

[2] B.A. Grierson, et al. Nuclear Fusion, 53(6):063010, June 2013.

[3] N. C. Luhmann, et al. Fusion Science and Technology, 53:335, 2008.

[4] H. Bindslev. J. Atmos. Terr. Phys., 58:983-989, 1996.

[5] A. J. H. Donné, et al. Nuclear Fusion, 47(6):S337-S384, June 2007.

[6] M. Salewski, et al. Nuclear Fusion, 50(3):035012, March 2010.

[7] S. K. Nielsen, et al. Nuclear Fusion, 51(6):063014, June 2011.

[8] R. Behn, et al. Physical Review Letters, 62(24):2833-2836, June 1989.

[9] E. V. Suvorov, et al. Plasma Physics and Controlled Fusion, 37(11):1207-1213, 1995.

[10] M Stejner, et al. Plasma Physics and Controlled Fusion, 55(8):085002, August 2013.

[11] M. Stejner, et al. Review of Scientific Instruments, 85(9):093504, September 2014.

[12] A. Herrmann and O. Gruber. FUSION SCIENCE AND TECHNOLOGY, 44(3):569 - 577, 2003.

[13] D. Wagner, et al. Nuclear Fusion, 48(5):054006, May 2008.

[14] J. Rasmussen, et al. arXiv:1310.2406, October 2013.

[15] H. Bindslev. Review of Scientific Instruments, 70(1):1093, 1999.

[16] W. A. Houlberg, et al. Physics of Plasmas, 4(9):3230, September 1997.

[17] A. Pankin, et al. Computer Physics Communications, 159(3):157-184, June 2004.

[18] Per Helander and Dieter J. Sigmar. Collisional transport in magnetized plasmas. Cambridge University Press, 2002. 